\title{
How to break the replica symmetry in structural glasses
}

\author{
V.G.Rostiashvili ${ }^{(a, b)}$, and T.A. Vilgis ${ }^{(a)}$ \\ (a) Max-Planck-Institut für Polymerforschung, Postfach 3148, D-55021 Mainz, Germany \\ (b) Chemical Physics, Russian Academy of Science, 142432, Chernogolovka, Moscow region, \\ Russia
}

\begin{abstract}
The variational principle (VP) has been used to capture the metastable states of a glass-forming molecular system without quenched disorder. It has been shown that VP naturally leads to a self-consistent random field GinzburgLandau model (RFGLM). In the framework of one-step replica symmetry breaking (1-RSB) the general solution of RFGLM is discussed in the vicinity of the spinodal temperature $T_{A}$ in terms of "hidden" formfactors $\tilde{g}(\mathbf{k}), g_{0}(\mathbf{k})$ and $\Delta(\mathbf{k})$. The self-generated disorder spontaneously arises. It is argued that at $T<T_{A}$ the activated dynamics is dominant.
\end{abstract}

PACS: 05.20.-y, 61.43-j, 61.43.Fs

Typeset using REVTEX 
It is a common knowledge that the transition from a liquid to a glass is mainly determined by extremely slow dynamic processes. On one hand, the mode coupling theory (MCT) predicts a pure kinetic transition temperature, $T_{c}$, where the density fluctuations are frozen and the system becomes nonergodic [1]. On the other hand, there is a strong trend in favour of the idea that the appearance of metastable states in the free energy landscape significantly determines the dynamical arrest at some transition temperature $T_{A}$ [2]. This consideration was based on both Potts [2, 4] and $p$ - spin [3] glass models, in which the quenched disorder was given. The authors drew the conclusion that these spin-glass models without reflection symmetry are also relevant for the structural glasses, where the disorder in a sense should not be quenched but self-generated [5].

Recently a great deal of work was done to incorporate the ideas and notions of the meanfield theory of the spin-glasses [6] into the theory of the structural glasses [7] [1]. The main underlying idea is related to the free-energy landscape. In order to quantify this landscape paradigm several copies ( or "real replicas") of the same system have been introduced. In doing so a reference replica $y$ is a typical configuration distributed with a Boltzmann - Gibbs measure, whereas a replica $x$ is affected by the influence of the replica $y$ through some short ranged attractive potential. It is assumed that for a fixed value of $y$, which plays the role of the quenched variables, the value of $x$ thermalized at the temperature of the experiment. The small positive coupling constant $\varepsilon$ is imposed which forces the variables $x$ to stay near the $y$ variables. If this correlation between $x$ and $y$ variables persists even at $\varepsilon \rightarrow 0$ the system is in a glassy phase. As a main result the expression for the "effective potential" has been calculated which shows a nontrivial solution for the "overlap" $q(x, y)$ [7].

In this letter we shall present a different general method in order to treat the metastable states in an arbitrary glass-forming molecular system without the quenched disorder. For this purpose we reduce the initial problem to a self-consistent random field Ginzburg-Landau model (RFGLM) by making use of the Feynman variational principle (VP). We shall show that the VP automatically yields the standard replica structure in phase space with the final limit $n \rightarrow 0$ for the number of replicas. This RFGLM is investigated by implementation of 
more or less standard field-theoretical tools [12 15]. The replica symmetry breaking (RSB) manifest itself as a $1^{\text {st }}$ order phase transition in the framework of a one-step RSB (1-RSB) scenario and the self-generated disorder shows up. Some comparison with the "real replica" method [7-11] will be given below.

We shall start with a low-molecular system of $N$ particles with coordinates $\mathbf{r}^{(p)},(p=$ $1, \ldots, N)$ via a pair potential $V(\mathbf{r})$. The partition function at temperature $T=1 / \beta$ is given by

$$
Z=\int \prod_{p=1}^{N} d^{3} \mathbf{r}^{(p)} \exp \left\{-\frac{\beta}{2} \sum_{p, m=1}^{N} V\left(\mathbf{r}^{(p)}-\mathbf{r}^{(m)}\right)\right\}
$$

A physically meaningful representation of eq. (1) in terms of the collective density, $\rho(\mathbf{r})=$ $\sum_{p=1}^{N} \delta\left(\mathbf{r}-\mathbf{r}^{(p)}\right)$, is given by

$$
Z=\int D \rho(1) \exp \left\{W[\rho]-\frac{\beta}{2} \int d 1 d 2 \rho(1) V(1-2) \rho(2)\right\},
$$

where the entropy of the system is related to the Jacobian of the corresponding transformation

$$
W[\rho]=\log \int \prod_{p=1}^{N} d 1^{(p)} \delta\left[\rho(1)-\sum_{p=1}^{N} \delta\left(1-1^{(p)}\right)\right]
$$

and the short - hand notation $\mathbf{r}^{(p)} \equiv 1^{(p)}$ has been used. An alternative appropriate representation of eq. (四) can be obtained in terms of a field $\psi(\mathbf{r})$ conjugated to $\rho(\mathbf{r})$ which appears as a result of the functional Fourier transformation

$$
\exp \{K[\psi]\}=\int D \rho(1) \exp \left\{W[\rho]-i \int d 1 \rho(1) \psi(1)\right\}
$$

where $K[\psi]$ is the cumulant generating functional for the free system. The functional expansion for $K[\psi]$ can be simply obtained in an explicit form. The invertion of eq. (4) and substitution in eq. (11) gives

$$
Z=\int D \psi \exp \left\{K[\psi]-\frac{1}{2} \int d 1 d 2 \psi(1)[\beta V]^{-1}(1-2) \psi(2)\right\}
$$

As a result, eqs. (2) and (5) provide two equivalent representations of the partition function. 
Our main objective in the following is to calculate the branch of the free energy which appears in a supercooled (below the melting point) liquid and which corresponds to the metastable states. To this end we recall that in a glassy phase only the component average free energy

$$
\bar{F}=F_{c}+T \Sigma
$$

is physically meanigful [16]. In eq. (6) the canonical free energy $F_{c}=-T \log Z$ and the function $\Sigma \geq 0$ is called the complexity [16]. On the other hand it is known that the Feynman VP, when implemented for the partition function (2), yields an upper limit for $F_{c}$ , i.e. $F_{c} \leq F_{\mathrm{VP}}$. As the basic assumption we take therefore

$$
\bar{F}=\min \left\{F_{0}+\left\langle\frac{\beta}{2} \int d 1 d 2 \rho(1) V(1-2) \rho(2)-W[\rho]-S[\rho]\right\rangle_{s}\right\}
$$

where the trial (Gaussian) Hamiltonian

$$
S[\rho]=\frac{1}{2} \int d 1 d 2\left\{G^{-1}(1,2) \rho(1) \rho(2)-2 G^{-1}(1,2) \rho(1)\langle\rho(2)\rangle_{s}\right\}
$$

Here the expectation value $\langle\rho(1)\rangle_{s}$ and the correlator $G(1,2) \equiv\langle\delta \rho(1) \delta \rho(2)\rangle_{s}$ are considered as independent variational functions. The assumption (7) means that the variational free energy $F_{\mathrm{VP}}$ takes its minimal value on the ensemble of components (or pure states). It will be justified a posteriori by the appearance of the replica structure. The minimization in eq. (7) with respect to $\langle\rho(1)\rangle_{s}$ and $G(1,2)$ leads to

$$
\begin{gathered}
\langle\rho(1)\rangle_{s}=\int d 2[\beta V]^{-1}(1,2)\left\langle\frac{\delta}{\delta \rho(2)} W[\rho]\right\rangle_{s}, \\
G(1,2)=\left[\beta V-\left\langle\frac{\delta^{2}}{\delta \rho(1) \delta \rho(2)} W[\rho]\right\rangle_{s}\right]^{-1}(1,2),
\end{gathered}
$$

where the "quenched" $\psi$ - expectation value reads

$$
\left\langle\frac{\delta}{\delta \rho(2)} W[\rho]\right\rangle_{s}=\left\langle\frac{\int D \psi i \psi(2) \exp \left\{K[\psi]+i \int d 1 \rho(1) \psi(1)\right\}}{\int D \psi \exp \left\{K[\psi]+i \int d 1 \rho(1) \psi(1)\right\}}\right\rangle_{s}
$$

and the "quenched" $\psi$ - correlator 


$$
\left\langle\frac{\delta^{2}}{\delta \rho(1) \delta \rho(2)} W[\rho]\right\rangle_{s}=-\left\langle\frac{\int D \psi \delta \psi(1) \delta \psi(2) \exp \left\{K[\psi]+i \int d 1 \rho(1) \psi(1)\right\}}{\int D \psi \exp \left\{K[\psi]+i \int d 1 \rho(1) \psi(1)\right\}}\right\rangle_{s}
$$

arises naturally in this representation. As a result we have reduced the problem to a selfconsistent RFGLM . We have used the term "quenched" because the Gaussian field $\rho(1)$ plays the role of an "external quenched" field in eqs.(11)- (12). Its moments however should eventually be determinated self-consistantly from eqs. (91)-(10).

One should not be surprised that Nature reveals the replica structure through VP. It is well known ( see e.g. Sec. 4 in [17]) that the rigorous treatment of statistical thermodynamics yields only thermal equilibrium states. In order to capture the metastable states in the framework of statistical thermodynamics one has to constrain properly the phase space of the considered system. The most famous example is the van der Waals - Maxwell metastable loop, which exists in the mean - field approximation and becomes flatter if the clusters formation are taken into account [17]. It is the VP in our case which implements this constraint.

The "quenched" moments (11)-(12) can be calculated by the standard way [6] from the replicated partition function

$$
\begin{aligned}
\left\langle Z^{n}\right\rangle_{s}\left\{G(1,2),\langle\rho(1)\rangle_{s}\right\} & =\int \prod_{a=1}^{n} D \psi_{a} \exp \left\{-\frac{1}{2} \int d 1 d 2 \sum_{a, b=1}^{n}\left[\rho_{0} \delta_{a b} \delta(1,2)+G(1,2)\right] \psi_{a}(1) \psi_{b}(2)\right. \\
& \left.+i \int d 1\langle\rho(1)\rangle_{s} \sum_{a=1}^{n} \psi_{a}(1)+\sum_{a=1}^{n} \tilde{K}\left[\psi_{a}\right]\right\}
\end{aligned}
$$

where $\rho_{0}$ is an average density and $\tilde{K}\left[\psi_{a}\right]$ is the anharmonic part of $K\left[\psi_{a}\right]$. As has been shown in ref. 12 15 the effective Hamiltonian such as in eq. (13) may lead to the replica symmetry breaking (RSB) which corresponds to a structural glass transition.

It is important to mention that for potentials $V(\mathbf{r})$ with the infinite range interactions we can directly expand the effective Hamiltonian in eq. (2) around the saddle point solution $\bar{\rho}(1)$ up to the second order. The calculations show that this next to the mean-field approximation and the VP merge and that both become exact, i.e. $\bar{F}=F_{c}$. In this case the glassy phase does not appear. This conclusion for the present realistic model (1) is in agreement with the 
result for a $\mathcal{O}(M)$ - model with a quartic interaction in the large $M$ limit [7] as well as for the particles on a hypersphere [18]. For the model given by eq. (11) with the infinite range of interaction the self-generated disorder is not generic and only crystallization [19] can be expected. It can be shown [20] also that the Langevin dynamics of the pure (i. e. without a quenched disorder ) system with the screened Coulomb potential $V(\mathbf{r})=(\mu / N) \exp (-\gamma r) / r$ ( where the interaction parameter $\mu>0$, the inverse screening length $\gamma \propto N^{-1 / 2}$ and the number of particles $N>>1$ ) does not reveal any glass transition. Despite ongoing discussions [21] the glassy dynamic behavior of the pure models is still debated.

Now we shall mention briefly the technical tools to calculate the free energy $\mathcal{F}\left\{\mathcal{G}_{a b}(1,2),\left\langle\psi_{a}(1)\right\rangle\right\}$ as a functional of the expectation value $\left\langle\psi_{a}(1)\right\rangle$ and the correlator $\mathcal{G}_{a b}(1,2)$ for the replicated field theory given by eq. (13). To this end, as in ref. [14, 15], we have used the second Legendre transformation. The detailed analysis shows that the Hartreelike contribution into the so-called generating functional of all 2-irreducible diagrams is zero. The first nontrivial contribution comes from the second-order terms in powers of vertices in eq. (13). The resulting free-energy functional $\mathcal{F}$ has been parametrized in the framework of the Parisi Anzatz [6]: $\mathcal{G}_{a b}(1,2) \longrightarrow\{\tilde{g}(\mathbf{k}), g(\mathbf{k}, x)\}$ where the formfactor $\tilde{g}(\mathbf{k})$ corresponds to the diagonal and $g(\mathbf{k}, x)$ to the off-diagonal elements of the hierarchical matrix $\mathcal{G}_{a b}(1,2)$. The wave vector $\mathbf{k}$ - dependence is retained in the spirit of ref. 22]. In 22] one can also find the beneficial formulas regarding the algebra of the hierarchical matrices. In the off-diagonal formfactor $g(\mathbf{k}, x)$ the argument $x \in[0,1]$.

We have restricted ourselves to the simplest case of 1-RSB scenario [6]. This means that the off-diagonal formfactor $g(\mathbf{k}, x)$ consists only of two pieces: $g(\mathbf{k}, x)=g_{0}(\mathbf{k})$ at $x<x_{c}$ and $g(\mathbf{k}, x)=g_{1}(\mathbf{k})$ at $x>x_{c}$, where $x_{c}$ is the break point. It has been shown before that for both Potts [2, 4] and $p$-spin glass models [3,23] the 1-RSB scenario is generic.

It is natural to define the gap $\Delta(\mathbf{k}) \equiv g_{1}(\mathbf{k})-g_{0}(\mathbf{k})$ as a non-ergodicity (order) parameter of the required glassy phase transition. As a consequence of these calculations the free-energy functional $\mathcal{F}\left\{\tilde{g}(\mathbf{k}), g_{0}(\mathbf{k}), \Delta(\mathbf{k})\right\}$ has been obtained. The extremization of it with respect to $\tilde{g}(\mathbf{k})$ and $g_{0}(\mathbf{k})$ at $\Delta(\mathbf{k})=0$ yields the equations for the replica symmetric $(\mathrm{RS})$ case 


$$
\begin{array}{r}
\frac{1}{\tilde{g}(\mathbf{k})-C(\mathbf{k})}-\frac{1}{\tilde{g}(\mathbf{k})-g_{0}(\mathbf{k})}+\frac{g_{0}(\mathbf{k})}{\left[\tilde{g}(\mathbf{k})-g_{0}(\mathbf{k})\right]^{2}}+\rho_{0} \\
+\frac{\rho_{0}^{2}}{2} \int_{\mathbf{k}_{1}} \tilde{g}\left(\mathbf{k}-\mathbf{k}_{1}\right) \tilde{g}\left(\mathbf{k}_{1}\right)-\frac{\rho_{0}^{2}}{6} \int_{\mathbf{k}_{1}, \mathbf{k}_{2}} \tilde{g}\left(\mathbf{k}-\mathbf{k}_{1}-\mathbf{k}_{2}\right) \tilde{g}\left(\mathbf{k}_{1}\right) \tilde{g}\left(\mathbf{k}_{2}\right)=0 \\
\frac{g_{0}(\mathbf{k})}{\left[\tilde{g}(\mathbf{k})-g_{0}(\mathbf{k})\right]^{2}}+\frac{1}{\tilde{g}(\mathbf{k})-C(\mathbf{k})}+\frac{\rho_{0}^{2}}{2} \int_{\mathbf{k}_{1}} g_{0}\left(\mathbf{k}-\mathbf{k}_{1}\right) g_{0}\left(\mathbf{k}_{1}\right) \\
-\frac{\rho_{0}^{2}}{6} \int_{\mathbf{k}_{1}, \mathbf{k}_{2}} g_{0}\left(\mathbf{k}-\mathbf{k}_{1}-\mathbf{k}_{2}\right) g_{0}\left(\mathbf{k}_{1}\right) g_{0}\left(\mathbf{k}_{2}\right)=0
\end{array}
$$

where $\int_{\mathbf{k}} \equiv \int d^{d} \mathbf{k} /(2 \pi)^{d}$ and we used the approximation $-\beta V(\mathbf{k})=C(\mathbf{k})(C(\mathbf{k})$ is the direct correlation function [24]). For simplicity we have consided the homogeneous case, $\langle\rho(1)\rangle_{s}=$ const, to eliminate the linear term with respect to $\psi$ in eq. (13).

In order to grasp the glassy (or 1-RSB) solution , $\mathcal{F}\left\{\tilde{g}, g_{0}, \Delta\right\}$ has been expanded up to the 4 -th order with respect to $\Delta(\mathbf{k})$ around the RS-solution (14). As a result the increment of the free energy connected with the non-zero order parameter $\Delta(\mathbf{k})$ is determined by the Landau expansion

$$
\begin{array}{r}
\mathcal{F}_{1}\{\Delta\}=-\int_{\mathbf{q}_{1}, \mathbf{q}_{2}} \Gamma_{2}\left(\mathbf{q}_{1}, \mathbf{q}_{2} ; x_{c}\right) \Delta\left(\mathbf{q}_{1}\right) \Delta\left(\mathbf{q}_{2}\right)-\int_{\mathbf{q}_{1}, \mathbf{q}_{2}, \mathbf{q}_{3}} \Gamma_{3}\left(\mathbf{q}_{1}, \mathbf{q}_{2}, \mathbf{q}_{3} ; x_{c}\right) \Delta\left(\mathbf{q}_{1}\right) \Delta\left(\mathbf{q}_{2}\right) \Delta\left(\mathbf{q}_{3}\right) \\
-\int_{\mathbf{q}_{1}, \mathbf{q}_{2}, \mathbf{q}_{3}, \mathbf{q}_{4}} \Gamma_{4}\left(\mathbf{q}_{1}, \mathbf{q}_{2}, \mathbf{q}_{3}, \mathbf{q}_{4} ; x_{c}\right) \Delta\left(\mathbf{q}_{1}\right) \Delta\left(\mathbf{q}_{2}\right) \Delta\left(\mathbf{q}_{3}\right) \Delta\left(\mathbf{q}_{4}\right)+\ldots
\end{array}
$$

The form of the expansion coefficients is determined by the RS-solution of eq. (14) as it will be given in an extended paper [25]. Since $\Gamma_{3}$ is generally speaking nonzero the transition is discontinuous. As in ref. 24 our free energy is proportional to the self-overlapping fraction : $\mathcal{F}_{1} \propto-\left(1-x_{c}\right)$. In this case, as has been argued in [2] 田, the metastable states appear first at the spinodal point $T_{A}$ which is determined by

$$
\lim _{x_{c} \rightarrow 1} \frac{\delta}{\delta \Delta(\mathbf{q})}\left\{\frac{\mathcal{F}_{1}\{\Delta\}}{1-x_{c}}\right\}_{\Delta=\bar{\Delta}}=0
$$

provided that the eigenvalues of the matrix

$$
\chi^{-1}\left(\mathbf{q}_{1}, \mathbf{q}_{2}\right)=-\lim _{x_{c} \rightarrow 1} \frac{\delta_{2}}{\delta \Delta\left(\mathbf{q}_{1}\right) \delta \Delta\left(\mathbf{q}_{2}\right)}\left\{\frac{\mathcal{F}_{1}\{\Delta\}}{1-x_{c}}\right\}_{\Delta=\bar{\Delta}}
$$


are nonnegative.

It is an open question whether the MCT transition temperature $T_{c}$ is related to $T_{A}$. For spin models with quenched disorder has been shown that these temperatures coincide [2] 4 . In any case at $T<T_{A}$ the dynamics is dominated by the activated process [2,26].

It is interesting that within the "hidden" correlators $\tilde{g}(\mathbf{k}), g_{0}(\mathbf{k})$ and $g_{1}(\mathbf{k})$ only $\tilde{g}(\mathbf{k})$ is related to the density correlator: $G(\mathbf{k})=[\tilde{g}(\mathbf{k})-C(\mathbf{k})]^{-1}$ (see eq. (10)), so that the discontinuous transition does not shows up through the density correlator $G(\mathbf{k})$ which is measured by an experiment. Nevertheless one can expect that below the spinodal point $T_{A}$ the activated dynamics becomes dominant and manifests itself in the time-dependent density correlator $G(\mathbf{k}, t)$. We shall return to this question in the near future. The equations for the RS-case (14) should be solved numerically first and then one can solve eq.(16)- (17) for the order parameter $\bar{\Delta}(\mathbf{k})$.

The glassy state appears first as a metastable one $\left(-\mathcal{F}_{1}\{\Delta\} \geq 0\right)$ but upon further cooling it can become stable: $-\mathcal{F}_{1}\{\Delta\} \leq 0$ (we recall that the conditions (16)- (17) determine the local maximum as usual in spin - glasses [6]). This possibility has been discussed in ref. [27] where on a basis of the Ramakrishnan - Yussouff free energy functional many inhomogeneous ( glassy ) density configurations have been found. On the contrary, in spin - glasses the glassy state is thermodynamically metastable for $T_{K}<T<T_{A}$ ( $T_{K}$ is the temperature where the complexity $\Sigma$ becomes non-extensive) [2, 4,26].

We would shortly like to compare our method with the mentioned "real replica" approaches [1]. The main difference is that in this method we deal with the conventional replicas where the "zero replica limit" and RSB are already fairly standard [6]. This leads, among other things, to a close link with the generalized spin-glass models results [2] 4,27]. Our consideration based on the well defined replicated field theory (see eq. (13) ) which needs as "an input" only information about the direct correlation function [19]. Conversely, in the "real replica" approach one has to choose one or other simple liquid models [24] (e.g. the replicated hypernetted chain (HNC) approximation in ref. [9, 10] ). Finally, our considerations can be directly generalized to the Langevin dynamics by making use of the 
MSR- generating functional method [20,28], whereas it is rather unclear what a dynamical counterpart of $\mathrm{HNC}$ in the simple liquid theory is.

In summary, we have suggested a general method to deal with the equilibrium structural glass transition. Making use of the VP naturally leads to the self-consistent RFGLM and the RSB - transition at some spinodal temperature $T_{A}$. In the framework of 1-RSB scenario the equations for the set of "hidden" formfactors $\tilde{g}(\mathbf{k}), g_{0}(\mathbf{k})$ and $\Delta(\mathbf{k})$ are derived. Although the static density correlator $G(\mathbf{k})$ does not show the RSB its dynamical counterpart could be considerably affected by this transition through the onset of the activated dynamics.

The authors have benefited from discussions with J.Baschnagel, K.Binder, W. Kob, A.Heuer , K.Müller-Nedebock and gratefully acknowledge financial support by the Sonderforschungsbereich 262 . 


\section{REFERENCES}

[1] W. Götze , in Liquids, Freezing and Glass Transition, Proceedings of the Les Houches Summer School, ed. by H.P. Hansen, D. Levesque and J. Zinn-Justin (North - Holland, Amsterdam, 1989)

[2] T.R. Kirkpatrik and D.P,G. Wolyness,Phys. Rev. B 36,8552 (1987)

[3] T.R. Kirkpatrik and D. Thirumalai,Phys. Rev. B 36,5388 (1987)

[4] T.R. Kirkpatrik and D. Thirumalai,Phys. Rev. B 37,5342 (1988)

[5] J.-P. Bouchaud, L.F. Cugliandolo, J. Kurchan and M.Mézard, in Spin Glasses and Random Fields, ed. by A.P. Young, Wold Scientific , 1997.

[6] M. Mézard, G. Parisi and M.A. Virazoro, Spin Glass and Beyond, Singapore, Wold Scientific, 1987.

[7] R. Monasson, Phys.Rev. Lett. 75, 2845 (1995)

[8] S. Franz and G. Parisi, Phys. Rev. Lett. 79 , 2486 (1997)

[9] M. Cardenas, S. Franz and G. Parisi J.Chem.Phys. 110, 1726 (1999)

[10] M. Mézard and G. Parisi, J.Phys. A 29,6515 (1996)

[11] M. Mézard and G. Parisi,Phys. Rev. Lett. 82, 747 (1999) ; M. Mézard , condmat/9812024

[12] M. Mézard and A.P. Young, Europhys. Lett.,18 , 653 (1992)

[13] M. Mézard and R. Monasson,Phys.Rev. B 50 , 7199 (1994)

[14] C. DeDominicis, H. Orland and T. Temesvari ,J.Phys.I (France) 5987 (1995)

[15] S. Stepanow, A.V. Dobrynin, T.A. Vilgis and K. Binder, J.Phys.I (France) 6837 (1996)

[16] R.G. Palmer, Adv.Phys.31 669 (1982) 
[17] K. Binder , Rep. Prog. Phys. 50, 783 (1987)

[18] L.F. Cugliandolo, J.Kurchan and G.Parisi, Phys. Rev.Lett. 741012 (1995)

[19] T.V. Ramakrishnan and M. Yussouff Phys.Rev. B 192775 (1979); D. Oxtoby in Liquids, Freezing and Glass Transition, Proceedings of the Les Houches Summer School, ed. by H.P. Hansen, D. Levesque and J. Zinn-Justin (North - Holland, Amsterdam, 1989)

[20] V.G. Rostiashvili and T.A. Vilgis ( in preparation )

[21] E. Marinari, G. Parisi and F. Retort,J.Phys. A 28327 (1995); S. Franz and J. Hertz , Phys.Rev.Lett.74 2114 (1995)

[22] M. Mézard and G. Parisi,J.Phys.I (France) 1, 809 (1991)

[23] E. Gardner, Nucl.Phys. B 254 [FS 14] 747 (1985); A. Crisanti and H.- J. Sommers, Z.Phys.B 87341 (1992)

[24] J.-P. Hansen and I.R. McDonald, Theory of simple liquids, Academic Press, 1990

[25] V.G. Rostiashvili and T.A. Vilgis ( in preparation )

[26] T.R. Kirkpatrik and D. Thirumalai and P.G. Wolyness, Phys. Rev. A 401045 (1989)

[27] C. Dasgupta, Europhys. Lett.,20 , 131 (1992); C. Dasgupta and S. Ramaswamy, Physica A 186, 314 (1992) ; C. Dasgupta, cond-mat/9808142

[28] V.G. Rostiashvili, M. Rehkopf and T.A. Vilgis, Eur. Phys. J. 6233 (1998) 